\title{
Minimal criteria for the diagnosis of avulsion of the puborectalis muscle by tomographic ultrasound
}

\author{
Hans Peter Dietz • Maria Jose Bernardo • \\ Adrienne Kirby $\cdot$ Ka Lai Shek
}

Received: 14 July 2010 /Accepted: 6 November 2010 /Published online: 24 November 2010

(C) The International Urogynecological Association 2010

\begin{abstract}
Introduction and hypothesis Puborectalis avulsion is a likely etiological factor for female pelvic organ prolapse (FPOP). We performed a study to establish minimal sonographic criteria for the diagnosis of avulsion.

Methods We analysed datasets of 764 women seen at a urogynecological service. Offline analysis of ultrasound datasets was performed blinded to patient data. Tomographic ultrasound imaging (TUI) was used to diagnose avulsion of the puborectalis muscle.

Results Logistic regression modelling of TUI data showed that complete avulsion is best diagnosed by requiring the three central tomographic slices to be abnormal. This finding was obtained in $30 \%$ of patients and was associated with symptoms and signs of FPOP $(P<0.001)$. Lesser degrees of trauma ('partial avulsion') were not associated with symptoms or signs of pelvic floor dysfunction.

Conclusions Complete avulsion of the puborectalis muscle is best diagnosed on TUI by requiring all three central slices to be abnormal. Partial trauma seems of limited clinical relevance.
\end{abstract}

Keywords 3-D ultrasound - Avulsion - Female pelvic organ prolapse $\cdot$ Pelvic floor $\cdot$ Puborectalis muscle $\cdot$ Ultrasound

H. P. Dietz $\cdot$ M. J. Bernardo · A. Kirby $\cdot$ K. L. Shek

Sydney Medical School Nepean,

Penrith, Australia

\section{H. P. Dietz $(\bowtie)$}

Obstetrics and Gynaecology, Sydney Medical School Nepean,

Nepean Hospital,

Penrith, NSW 2750, Australia

e-mail: hpdietz@bigpond.com

$\begin{array}{ll}\text { Abbreviations } & \\ 3-D & \text { Three-dimensional } \\ \text { ICS POP-Q } & \begin{array}{l}\text { International Continence Society } \\ \text { Prolapse Quantification } \\ \text { PFMC }\end{array} \\ \text { Pelvic floor muscle contraction } \\ \text { ROI } & \text { Region of Interest } \\ \text { TUI } & \text { Tomographic ultrasound imaging }\end{array}$

Introduction

Major morphological abnormalities of the puborectalis muscle ('avulsion') are likely to be an etiological factor in the development of female pelvic organ prolapse $[1,2]$. It is clearly related to vaginal childbirth $[3,4]$, with Forceps delivery, length of second stage and foetal size as likely risk factors $[5,6]$. Such trauma is associated with abnormal muscle biometry and function as well as female pelvic organ prolapse [1, 2, 7-9]. Avulsion increases the area of the levator hiatus $[5,7]$, the largest potential hernial portal in the abdominal envelope. It has been described on magnetic resonance imaging [10], on ultrasound [11], intraoperatively [4, 12], and in the cadaver [13]. Avulsion may also be a predictor of prolapse recurrence [14, 15], implying that identification of women with avulsion may be of importance for pelvic reconstructive surgery. While this condition can be diagnosed by palpation, this requires substantial training [16, 17]. Diagnosis by magnetic resonance imaging and pelvic floor ultrasound is highly repeatable $[1,18]$.

One of the main current issues in the diagnosis of trauma to the puborectalis muscle is the distinction between partial and complete trauma, that is, the decision as to whether any part of the muscle is still attached to the inferior pubic 
ramus or not. Partial trauma is not uncommon, but to date, there is no data on its prevalence or clinical significance. Diagnosis requires assessment of the entire muscle, whether by palpation or by imaging. In the case of imaging, this implies the need for multislice or tomographic imaging in order to reduce the likelihood of small remnants of the muscle being missed. We therefore performed a retrospective study using 4-D ultrasound to establish minimal sonographic criteria for the diagnosis of complete avulsion and correlate the diagnosis of partial avulsion with symptoms and signs of pelvic organ prolapse and bladder dysfunction in a large cohort of women.

\section{Materials and methods}

This is a retrospective study of 764 women referred to a tertiary urogynecological service for symptoms of prolapse or lower urinary tract dysfunction from May 2005 to November 2008. All of them had undergone an interview, clinical assessment using the International Continence Society Prolapse Quantification (ICS POP-Q) system for prolapse grading, and 4-D translabial ultrasound. The ultrasound examination was performed supine after bladder emptying on maximum valsalva and on pelvic floor muscle contraction (PFMC). Blinded offline analysis of volume datasets was performed at least 6 months after the original patient contact using proprietary software (4D View v 7.0, General Electric Medical Ultrasound, Zipf, Austria) blinded to all patient data. Tomographic ultrasound imaging (TUI) was performed on volumes obtained at PFMC at $2.5-\mathrm{mm}$ slice intervals, from $5 \mathrm{~mm}$ below to $12.5 \mathrm{~mm}$ above the plane of minimal hiatal dimensions, and was used for assessment for puborectalis avulsion as previously described [19]. A PFMC was identified by a cranioventral lift of the bladder neck, with shortening of the anteroposterior diameter of the levator hiatus. Rest volumes were used in patients unable to perform a PFMC $(n=34)$.

The two lowermost TUI slices in a standard set of eight (i.e. those below the plane of minimal dimensions) were excluded from the analysis on the basis of a previous study showing that the appearance of avulsion in those slices may be artefactual due to the curved nature of the levator plane [20]. This leaves six slices on the right and six on the left side, i.e. a total of 12 , for analysis, reaching from the plane of minimal dimensions to $12.5 \mathrm{~mm}$ above this plane. To define avulsion in any given slice, we use the term 'abnormal insertion' rather than 'absence of muscle' on ultrasound imaging since it is not uncommon to see scar tissue connecting the retracted muscle to the sidewall, lateral to the original insertion, which could be misinterpreted as 'attachment'. In doubtful cases, we utilise the 'levator urethra gap (LUG) measurement' [21], which is a sonographic equivalent to the 'levator symphysis gap' [22] used on MR imaging. An insertion is regarded as abnormal when the LUG is over $2.5 \mathrm{~cm}$ as illustrated in Fig. 1.

Logistic regression analysis was performed in order to determine the optimal relationship between symptoms of prolapse/signs of significant prolapse (ICS POP-Q stage 2+) and partial avulsion on tomographic ultrasound. Since rightand left-sided abnormalities are not independent of each other, we performed the analysis separately for left and right. As it is inadmissible to treat single slices as independent predictors in the same model due to co-linearity, we created dummy variables by adding additional slices to the original model of any two slices out of the central three. Partial avulsion was diagnosed when any of slices three to eight were abnormal, without the patient being classified as a complete avulsion due to all three central slices being abnormal (see Fig. 1 for an example).

This study was approved by the local human research ethics committee (Sydney West Area Health Service Human Research Ethics Committee reference 05-029) as an extension of a previously approved project. Statistical analysis was undertaken using Minitab V. 13 (Minitab Inc., State College, PA, USA) and SPSS V 17 (SPSS Inc., Chicago IL, USA) for logistic regression modelling. Normality testing was performed using the KolmogorovSmirnov method. We used $t$ tests for comparison of normally distributed continuous data. A $P<0.05$ was taken to indicate statistical significance.

\section{Results}

The mean age was 55 (range, 18-89). Eighty-nine percent were vaginally parous $(n=682)$, and the median number of vaginal deliveries was 2 (range, $0-10$ ). One hundred eightysix (24\%) had had a vaginal operative delivery. Thirty-three percent reported a previous hysterectomy $(n=251)$. They complained of symptoms of stress urinary incontinence $(n=$ $541,71 \%)$, urge incontinence $(n=524,69 \%)$, frequency $(n=$ $399,52 \%)$, nocturia $(n=384,50 \%)$ and symptoms of prolapse $(n=337,44 \%)$. On examination, 50\% $(n=379)$ were shown to have significant pelvic organ prolapse (ICS POP-Q stage 2 or higher). This was a cystocele in $37 \%$ ( $n=$ $281)$, uterine prolapse in $7 \%(n=48)$ and a rectocele in $28 \%$ $(n=207)$. Of 764 patients, two volume datasets were not located, and two more were impossible to assess completely due to technical inadequacy, leaving 760 ultrasound volume datasets for later analysis. Another seven patients could not be examined due to vaginal stenosis or refusal, leaving 753 datasets for the analysis of the relationship between significant prolapse (ICS POP-Q stage 2 or higher) and TUI findings. Per patient, 12 slices were scored as normal or abnormal, equalling a total of 9,120 assessments. 
Fig. 1 Large bilateral partial trauma (indicated by asterisk) sparing the inferior aspects of the insertions of the puborectalis muscle (arrows). The numbers indicate the location of the slice relative to the reference plane (the plane of minimal hiatal dimensions, identified by ' 0 '). The numerical measurements (bottom right hand corner) give the 'levator-urethra gap' [21] which is useful in doubtful cases. A measurement of over $2.5 \mathrm{~cm}$ is regarded as abnormal

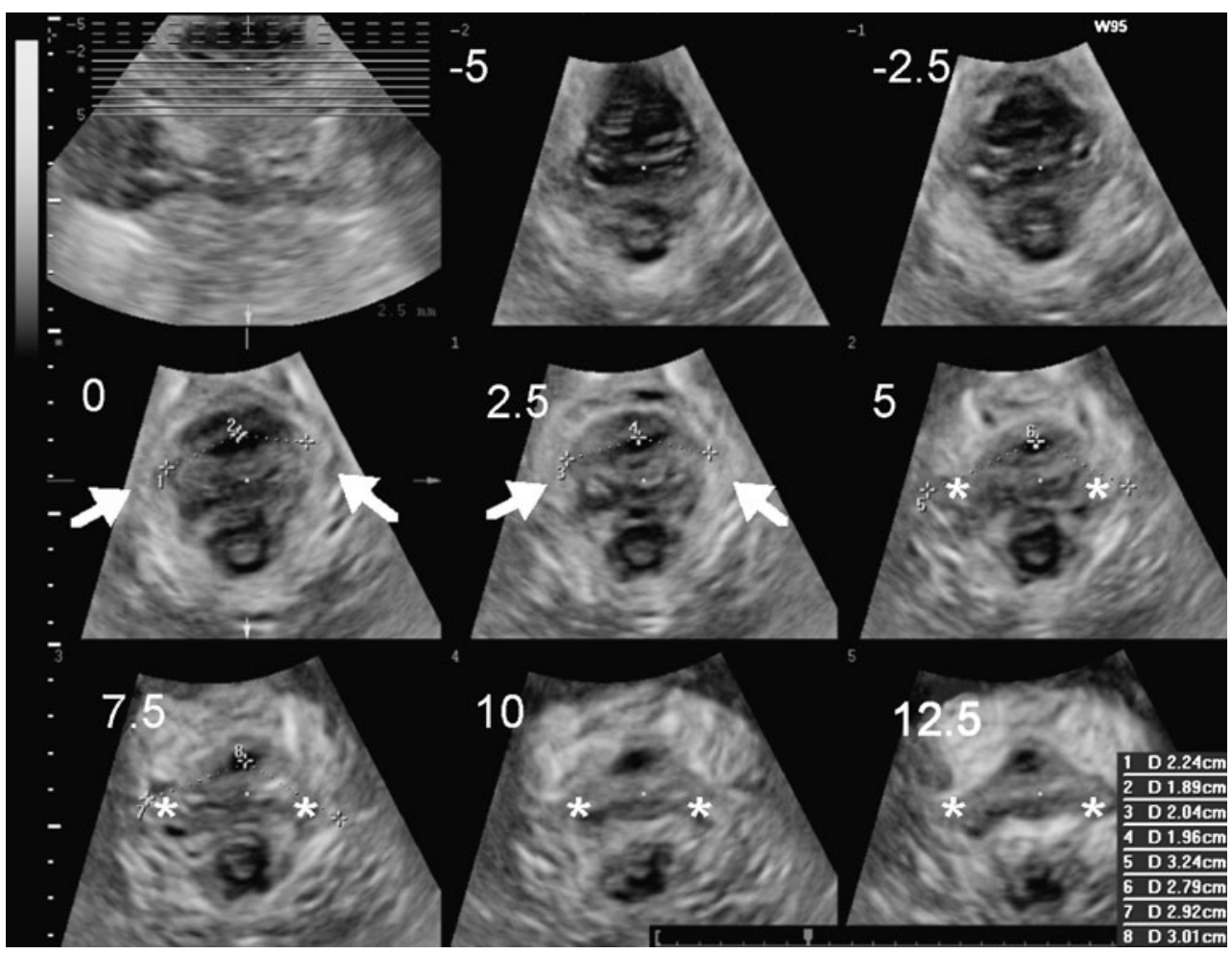

The results of logistic regression analysis performed to establish minimal sonographic criteria for the diagnosis of complete avulsion are shown in Tables 1 and 2. Table 1 shows the performance of different models for predicting symptoms of prolapse. In agreement with clinical experience, the relationship was not linear. Optimal prediction was achieved by a model including all three central slices, i.e. the slice at the plane of minimal dimensions plus the two above. Any two of the three central slices did not predict either outcome, and no extra information was gained by accounting for extra slices. This was true for both the left and the right sides. On performing the analysis for objective findings of significant prolapse, this pattern was repeated (see Table 2).

Using those minimal sonographic criteria, we found a complete avulsion in $30 \%$ of all patients $(n=226)$. It was right-sided (see Fig. 2 for an example) in $26 \%(n=199)$, left-sided in $20 \%(n=146)$ and bilateral in $16 \%(n=119)$. On TUI, 1,233 slices were positive on the right, and 1,024 were positive on the left. As shown previously [1], a complete avulsion was strongly associated with symptoms of prolapse $(59 \%$ vs $38 \%, P<0.001)$, significant prolapse on clinical assessment $(74 \%$ vs $40 \%, P<0.001)$ and cystocele descent on ultrasound $(14.8 \mathrm{~mm}$ below the symphysis vs. $2.3 \mathrm{~mm}$ below the symphysis, $P<0.001$; see Table 3).

After exclusion of patients with complete (unilateral or bilateral) avulsion ( $n=226)$ according to the criteria so established, 55 patients with TUI abnormalities who did not meet these minimal criteria were identified and classified as 'partial avulsion'. There was no association between partial avulsion and symptoms of bladder dysfunction (i.e. symptoms of incontinence, frequency, nocturia or voiding dysfunction) or prolapse, nor did we find an association between significant
Table 1 Results of logistic regression modelling of findings on tomographic ultrasound against symptoms of prolapse $(n=760)$

\begin{tabular}{|c|c|c|c|c|}
\hline \multirow[t]{3}{*}{ Model $(n=760)$} & \multicolumn{4}{|c|}{ Effect of slices involved versus no involvement } \\
\hline & \multicolumn{2}{|l|}{ Left } & \multicolumn{2}{|l|}{ Right } \\
\hline & OR $(95 \% \mathrm{CI})$ & $P$ value & OR $(95 \% \mathrm{CI})$ & $P$ value \\
\hline Any two of central three slices & $1.43(0.62-3.30)$ & 0.40 & $0.92(0.43-1.99)$ & 0.83 \\
\hline All central three slices & $2.30(1.64-3.43)$ & $<0.001$ & $2.49(1.71-3.63)$ & $<0.001$ \\
\hline All central three plus next above & $2.37(1.67-3.36)$ & $<0.001$ & $2.43(1.66-3.56)$ & $<0.001$ \\
\hline All central three plus next two above & $2.35(1.65-3.35)$ & $<0.001$ & $2.53(1.72-3.72)$ & $<0.001$ \\
\hline All central three plus next three above & $2.35(1.65-3.35)$ & $<0.001$ & $2.52(1.71-3.71)$ & $<0.001$ \\
\hline
\end{tabular}


Table 2 Results of logistic regression modelling of findings on tomographic ultrasound against significant objective prolapse (ICS POP-Q stage 2 or higher, $n=753$ )

\begin{tabular}{llllll}
\hline Model $(n=753)$ & \multicolumn{2}{l}{ Effect of slices involved versus no involvement } \\
\cline { 2 - 4 } & Left & & \multicolumn{2}{l}{ Right } & \\
\cline { 2 - 3 } \cline { 5 - 6 } & OR $(95 \% \mathrm{CI})$ & $P$ value & & OR $(95 \% \mathrm{CI})$ & $P$ value \\
\hline Any two of central three slices & $1.99(0.84-4.75)$ & 0.12 & & $1.18(0.56-2.49)$ & 0.66 \\
All central three slices & $3.46(2.41-4.98)$ & $<0.001$ & & $3.94(2.60-5.97)$ & $<0.001$ \\
All central three plus next above & $3.62(2.49-5.26)$ & $<0.001$ & & $3.98(2.60-6.09)$ & $<0.001$ \\
All central three plus next two above & $3.74(2.55-5.49)$ & $<0.001$ & $3.88(2.53-5.95)$ & $<0.001$ \\
All central three plus next three above & $3.39(1.05-10.97)$ & $<0.001$ & $3.90(2.54-5.98)$ & $<0.001$ \\
\hline
\end{tabular}

prolapse on ICS POP-Q and partial avulsion (see Table 3). There was some evidence of greater cystocele descent on ultrasound in women with partial avulsion (maximum descent to -6.0 below the symphysis pubis vs. -1.9 below in patients with normal TUI; $P=0.12$ on $t$ test).

\section{Discussion}

In this large retrospective series of symptomatic women attending a tertiary urogynecological service, we tried to establish minimal sonographic criteria for the diagnosis of complete avulsion of the puborectalis muscle, and to define the clinical relevance of partial puborectalis avulsion for symptoms and signs of pelvic floor dysfunction. For this purpose, a total of 9,120 single slices were assessed at the plane of minimal dimensions and up to $12.5 \mathrm{~mm}$ above this plane, at $2.5-\mathrm{mm}$ slice intervals, as previously described [19].
Complete avulsion of the insertion of the puborectalis muscle from the inferior pubic ramus seems to be of importance for pelvic organ support, especially for anterior and central compartment descent, and this was again confirmed in this series. Several different methodologies have been proposed, of which multislice or tomographic ultrasound may be the most practical and repeatable [19]. This study provides, for the first time, objective data allowing us to optimise diagnostic algorithms based on tomographic ultrasound findings. Clinically, it appears that significant trauma is defined by a complete absence of muscle on the inferior pubic ramus, which by definition would include the entire puborectalis muscle. The imaging equivalent of such palpatory findings seems to be an abnormal muscle insertion in at least three tomographic slices obtained at the level of the plane of minimal dimensions as well as 2.5 and $5 \mathrm{~mm}$ above. In the present study, we have shown that fewer than three abnormal central slices are much less likely to be associated with
Fig. 2 Complete right-sided trauma (left on image, indicated by asterisk) affecting all tomographic slices from $5 \mathrm{~mm}$ below to $12.5 \mathrm{~mm}$ above the plane of minimal dimensions. The latter is identified by ' $O$ '

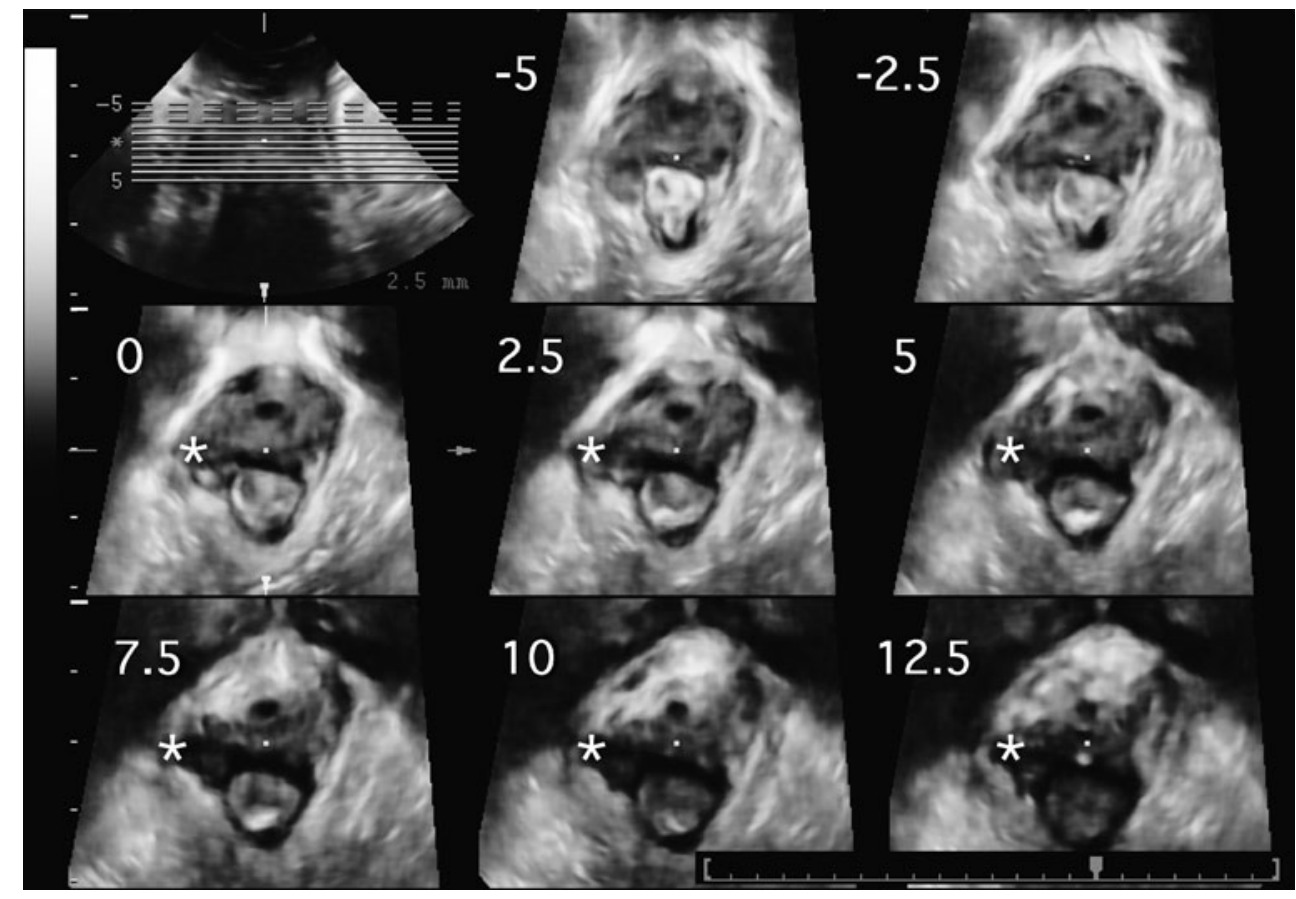


Table 3 The association between symptoms and signs of prolapse and complete/partial avulsion

\begin{tabular}{llllll}
\hline & $\begin{array}{l}\text { Complete avulsion } \\
N=226\end{array}$ & $\begin{array}{l}\text { All others } \\
N=534\end{array}$ & $P$ value & $\begin{array}{l}\text { Partial avulsion } \\
N=55\end{array}$ & $\begin{array}{l}\text { No avulsion } \\
N=479\end{array}$ \\
\hline Symptoms of prolapse & $133 / 226(59 \%)$ & $202 / 534(38 \%)$ & $<0.001$ & $18 / 55(33 \%)$ & $183 / 479(38 \%)$ \\
ICS POP-Q stage 2+ US & $164 / 223(74 \%)$ & $213 / 530(40 \%)$ & $<0.001$ & $24 / 55(44 \%)$ & $189 / 475(40 \%)$ \\
Cystocele descent & $-14.8($ SD 19.9$)$ & $-2.3($ SD 17.2$)$ & $<0.001$ & -6 (SD 18.6) & $-1.9($ SD 17) \\
\hline
\end{tabular}

Numbers vary due to missing data ( $n=7$ for ICS POP-Q assessment, $n=11$ for ultrasound prolapse assessment)

symptoms and signs of prolapse. Requiring more cranial slices to be positive does not improve model performance. Consequently, we propose that an abnormality in the three central slices, i.e. slices at the level of the plane of minimal dimensions and at 2.5 and $5 \mathrm{~mm}$ above this plane, be required as minimal diagnostic criterion for the diagnosis of a complete avulsion of the puborectalis muscle on tomographic pelvic floor ultrasound.

While the relatively low number of partial avulsions may not have provided sufficient power to identify minor effects, it seems reasonable to conclude that defects that do not meet these minimal critieria are probably not strongly associated with symptoms or signs of pelvic floor dysfunction. This may be due to the minor nature of such defects; however, it is also possible that some of those minor 'defects' may be artefactual in nature. If at least one of the three central levels (i.e. those that seem to represent puborectalis in every woman, irrespective of personal biometry) is still intact on tomographic ultrasound, the state of other levels (cranial or caudal) does not seem to be of great importance.

This is highly relevant in clinical practice as partial trauma is not uncommon (55/760 or $7 \%$ in this series) and may pose diagnostic difficulties. Both on palpation and on imaging, it is our experience that once practitioners have learned to identify avulsion, they are likely to progress towards over-diagnosis, identifying less obvious forms of trauma. Over-diagnosis may also occur on imaging where one level may show severe bilateral trauma while another seems entirely intact (see Fig. 1). An accurate assessment clearly requires multislice imaging.

An alternate method of diagnosing avulsion on pelvic floor ultrasound requires the use of rendered volumes, that is, semitransparent volume data rendered caudally to cranially [11]. In this method, the location of the caudal margin of the region of interest (ROI) or rendered volume will determine appearances, and in a patient with partial trauma, one may obtain rendered images that are normal or severely abnormal, depending on ROI placement. Palpation can be of help in interpreting imaging findings, especially to confirm completeness of trauma. Digital examination may detect remnants of muscle that were missed on imaging, and this may explain why, on average, imaging diagnoses more avulsions than palpation [23]. However, palpation requires a longer learning curve and is probably less repeatable [16, 17].

In conclusion, it seems prudent to require multislice imaging for the diagnosis of avulsion of the puborectalis muscle and to use clearly defined minimal criteria. We propose to diagnose a complete avulsion on tomographic pelvic floor ultrasound if all three central slices, i.e. the plane of minimal dimensions plus slices $2.5 \mathrm{~mm}$ and $5 \mathrm{~mm}$ cranial to this plane, show an abnormal insertion of the puborectalis muscle on the inferior pubic ramus. For the time being, partial avulsion can probably be ignored in clinical practise, although it may well be a marker for other forms of trauma and although it may matter in the individual patient. The relative value of palpation and imaging in the diagnosis of avulsion remains to be defined.

Conflicts of interest Dr. Dietz has acted as a consultant for American Medical Systems (Minnetonka, MN), Continence Control Systems (Sydney) and Materna Inc. (San Francisco, CA), accepted speaker's fees from General Electric Medical Ultrasound (Sydney, Australia), American Medical Systems, and Astellas (Tokyo, Japan), and has benefitted from equipment loans provided by General Electric, Bruel and Kjaer (Gentofte, Denmark), and Toshiba (North Ryde, Australia). Drs. Ka Lai Shek, Maria Jose Bernardo and Adrienne Kirby have no conflict of interest to disclose.

\section{References}

1. Dietz H, Simpson J (2008) Levator trauma is associated with pelvic organ prolapse. Br J Obstet Gynaecol 115:979-984

2. DeLancey J, Morgan D, Fenner D, Kearney R, Guire K, Miller J et al (2007) Comparison of levator ani muscle defects and function in women with and without pelvic organ prolapse. Obstet Gynecol 109:295-302

3. Dietz H, Lanzarone V (2005) Levator trauma after vaginal delivery. Obstet Gynecol 106:707-712

4. Dietz H, Gillespie A, Phadke P (2007) Avulsion of the pubovisceral muscle associated with large vaginal tear after normal vaginal delivery at term. Aust NZ J Obstet Gynaecol 47:341-344

5. Shek K, Dietz H (2009) The effect of childbirth on hiatal dimensions: a prospective observational study. Obstet Gynecol 113:1272-1278 
6. Valsky DV, Lipschuetz M, Bord A, Eldar I, Messing B, HochnerCelnikier D et al (2009) Fetal head circumference and length of second stage of labor are risk factors for levator ani muscle injury, diagnosed by 3-dimensional transperineal ultrasound in primiparous women. Am J Obstet Gynecol 201:91.e91-91.e97

7. Abdool Z, Shek K, Dietz H (2009) The effect of levator avulsion on hiatal dimensions and function. Am J Obstet Gynecol 201:89. e81-89.e85

8. Dietz HP, Shek C (2008) Levator avulsion and grading of pelvic floor muscle strength. Int Urogynecol J 19:633-636

9. Dietz HP, Steensma AB (2006) The prevalence of major abnormalities of the levator ani in urogynaecological patients. $\mathrm{Br}$ J Obstet Gynaecol 113:225-230

10. DeLancey JO, Speights SE, Tunn R, Howard D, Ashton Miller JA (1999) Localized levator ani muscle abnormalities seen in MR images: site, size and side of occurrence. Int Urogynecol J 10:S20-S21

11. Dietz H (2004) Ultrasound imaging of the pelvic floor: 3D aspects. Ultrasound Obstet Gynecol 23:615-625

12. Shobeiri S, Chimpiri A, Allen AC, Nihira M, Quiroz LH (2009) Surgical reconstitution of a unilaterally avulsed symptomatic puborectalis muscle using autologous fascia lata. Obstet Gynecol $114: 480-482$

13. Wallner C, Wallace C, Maas C, Lange M, Lahaye M, Moayeri N et al (2009) A high resolution 3D study of the female pelvis reveals important anatomical and pathological details of the pelvic floor. Neurourol Urodyn 28:668-670

14. Dietz HP, Chantarasorn V, Shek KL (2010) Levator avulsion is a risk factor for cystocele recurrence. Ultrasound Obstet Gynecol $36: 76-80$
15. Model A, Shek KL, Dietz HP (2010) Levator defects are associated with prolapse after pelvic floor surgery. Eur J Obstet Gynecol Reprod Biol. doi:10.1016/j.ejogrb.2010.07.046

16. Dietz HP, Shek KL (2008) Validity and reproducibility of the digital detection of levator trauma. Int Urogynecol J 19:10971101

17. Kearney R, Miller JM, Delancey JO (2006) Interrater reliability and physical examination of the pubovisceral portion of the levator ani muscle, validity comparisons using MR imaging. Neurourol Urodyn 25:50-54

18. Weinstein MM, Pretorius D, Nager CW, Mittal R (2007) Interrater reliability of pelvic floor muscle imaging abnormalities with 3D ultrasound. Ultrasound Obstet Gynecol 30:538

19. Dietz H (2007) Quantification of major morphological abnormalities of the levator ani. Ultrasound Obstet Gynecol 29:329334

20. Dietz HP, Shek K (2009) Tomographic ultrasound of the pelvic flooor: which levels matter most? Ultrasound Obstet Gynecol 33:698-703

21. Dietz H, Abbu A, Shek K (2008) The levator urethral gap measurement: a more objective means of determining levator avulsion? Ultrasound Obstet Gynecol 32:941-945

22. Singh K, Jakab M, Reid W, Berger LA, Hoyte L (2003) Threedimensional magnetic resonance imaging assessment of levator ani morphologic features in different grades of prolapse. Am J Obstet Gynecol 188:910-915

23. Moegni F, Shek K, Dietz H (2010) Diagnosis of levator avulsion injury: a comparison of three methods. Int Urogynecol J 21:S215S216 